STRUCTURAL BIOLOGY COMMUNICATIONS

ISSN 2053-230X

\section{Natural and non-natural amino-acid side-chain substitutions: affinity and diffraction studies of meditope-Fab complexes}

\author{
Krzysztof P. Bzymek, Kendra A. Avery,‡ Yuelong Ma, David A. Horne and \\ John C. Williams*
}

Received 31 August 2016

Accepted 11 October 2016

Edited by R. L. Stanfield, The Scripps Research Institute, USA

₹ Current address: Xencor, 111 West Lemon Avenue, Monrovia, CA 91016, USA.

Keywords: meditope; monoclonal antibody; cetuximab; unnatural amino acids; X-ray crystallography; surface plasmon resonance.

PDB references: cetuximab Fab, complex with CQYDLSTRRLKC meditope, 5t1 m; with CQHDLSTRRLKC meditope, 5euk; with GQQDLSTRRLKG meditope, $5 \mathrm{i} 2 \mathrm{i}$; with GQ(2-Br-F)DLSTRRLKG meditope, 5itf; with GQ(3-Br-F)-DLSTRRLKG meditope, 5ir1; with GQ(4-Br-F)DLSTRRLKG meditope, 5iop; with $\mathrm{CQA}(\mathrm{Ph})_{2}$ DLSTRRLKC meditope, 5t1l; with CQFDYSTRRLKC meditope, 5f88; with CQFDESTRRLKC meditope, 5etu; with CQFDQSTRRLKC meditope, 5th2; with CQFDA(Ph) ${ }_{2}$ STRRLKC meditope, 5t1k; with GQFDLST(Cit)RLKG meditope, 5ivz; with GQFDLSTR(Cit)LKG meditope, 5iv2; with CQFDLSTRRQKC meditope, $5 \mathrm{ff} 6$

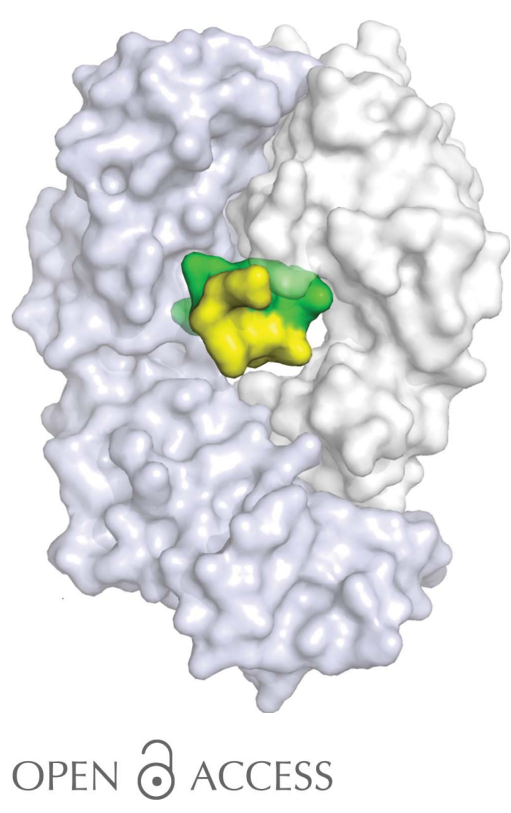

Department of Molecular Medicine, Beckman Research Institute of City of Hope, 1710 Flower Street, Duarte, CA 91010 , USA. *Correspondence e-mail: jcwilliams@coh.org

Herein, multiple crystal structures of meditope peptide derivatives incorporating natural and unnatural amino acids bound to the cetuximab Fab domain are presented. The affinity of each derivative was determined by surface plasmon resonance and correlated to the atomic structure. Overall, it was observed that the hydrophobic residues in the meditope peptide, Phe3, Leu5 and Leu10, could accommodate a number of moderate substitutions, but these invariably reduced the overall affinity and half-life of the interaction. In one case, the substitution of Phe3 by histidine led to a change in the rotamer conformation, in which the imidazole ring flipped to a solvent-exposed position. Based on this observation, Phe3 was substituted by diphenylalanine and it was found that the phenyl rings in this variant mimic the superposition of the Phe3 and His3 structures, producing a moderate increase, of 1.4-fold, in the half-life of the complex. In addition, it was observed that substitution of Leu5 by tyrosine and glutamate strongly reduced the affinity, whereas the substitution of Leu5 by diphenylalanine moderately reduced the half-life (by approximately fivefold). Finally, it was observed that substitution of Arg8 and Arg9 by citrulline dramatically reduced the overall affinity, presumably owing to lost electrostatic interactions. Taken together, these studies provide insight into the meditope-cetuximab interaction at the atomic level.

\section{Introduction}

Monoclonal antibodies (mAbs) are used clinically to treat a number of diseases, including cancer, arthritis and Crohn's disease (Emmons \& Hunsicker, 1987; Suzuki et al., 2015; Weiner et al., 2012). Owing to their specificity and favorable pharmacological properties, there is significant interest in adding new functionality (e.g. potent cytotoxins) to increase their efficacy and potency (Trail \& Bianchi, 1999; Wu \& Senter, 2005; Chari, 2008; Ducry \& Stump, 2010). Multiple strategies have been developed to modify monoclonal antibodies, including chemical conjugation (e.g. the introduction the introduction of an FGE site; Rabuka et al., 2012). Recently, we identified a cyclic, 12-amino-acid peptide that binds in a cavity formed by all four $\mathrm{IgG}$ domains of the cetuximab Fab (Fig. 1; Donaldson et al., 2013). Based on our observations, the peptide-Fab interaction does not affect antigen binding (Donaldson et al., 2013). Furthermore, we have demonstrated that we could graft the binding site onto antigen-binding properties (Donaldson et al., 2013). Owing to of cysteine; Bhakta et al., 2013) and protein engineering (e.g. other Fabs, including trastuzumab, without affecting their 
the location of the binding site within the Fab, we have termed the peptide a meditope (medi-, middle, + -tope, place). To exploit this interaction as a potential 'receptor' within the Fab to rapidly add functionality to cetuximab and grafted mAbs without the need for covalent modification of the antibody, we have created a number of meditope variants, solved their structures and measured their affinity by surface plasmon resonance (SPR). In a previous manuscript, we focused on different cyclization strategies of the meditope peptide (Bzymek et al., 2016). In this report, we focus on the modification of side chains that participate in the meditope-Fab interaction.

\section{Materials and methods}

All reagents were obtained from Sigma or Fisher. Peptides were synthesized at the Synthetic and Biopolymer Chemistry Core, City of Hope, Duarte, California, USA or by CS Bio Co., Menlo Park, California, USA. The Leu5Tyr variant was biosynthesized as described elsewhere (Donaldson et al., 2013). Fab purification, crystallization and structure solution were conducted as described previously (Bzymek et al., 2016). Briefly, for each complex, the Fab was mixed with excess peptide (1:10-1:15 molar ratio of Fab:peptide). The precipitant solution was composed of $0.1 M \mathrm{Na}_{2} \mathrm{HPO}_{4}, 0.1 M$ citric acid, 0.4-0.5 $M \mathrm{~K}_{2} \mathrm{HPO}_{4}, 1.6-1.8 M \mathrm{NaH}_{2} \mathrm{PO}_{4}$. Crystals were grown by vapour diffusion using the hanging-drop method at $20^{\circ} \mathrm{C}$ by mixing $1 \mu \mathrm{l}$ protein complex solution with $1 \mu \mathrm{l}$ precipitant solution. X-ray diffraction data were collected on a Rigaku

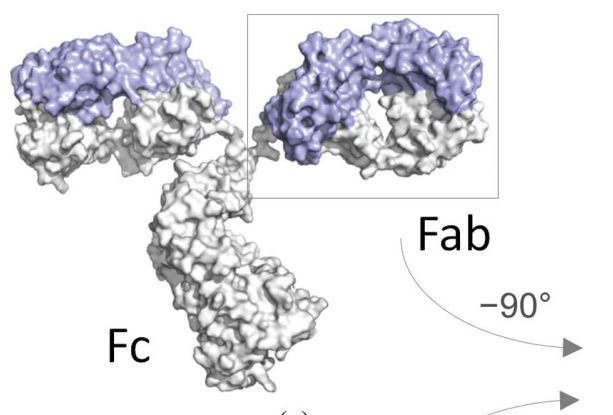

(a)

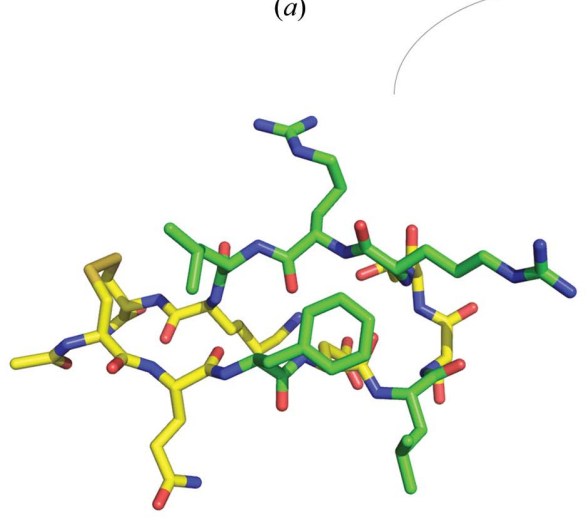

(b)
MicroMax-007 HF with an R-AXIS IV $^{++}$detector (at a wavelength of $1.5418 \AA$ ) or on SSRL beamline 12-2 (L5Q; PDB entry 5th2; at a wavelength of $1.0000 \AA$ ). SPR experiments were performed on a GE Biacore T100 instrument (GE Healthcare). Briefly, cetuximab IgG was immobilized on a CM5 chip using amine-coupling chemistry at a density suitable for kinetics experiments with peptide analytes ( $\sim 5000 \mathrm{RU})$. Peptides were dissolved in $0.5 \mathrm{ml}$ water and dialyzed with two changes against $500 \mathrm{ml}$ water to remove excess TFA. Quantification of peptide concentration was performed as described previously (Bzymek et al., 2016).

Analytes were passed over the chip at $30 \mu \mathrm{min}^{-1}$ in HBSEP+ [10 $\mathrm{m} M$ HEPES pH 7.4, $150 \mathrm{~m} M \mathrm{NaCl}, 3 \mathrm{~m} M$ EDTA, $0.05 \%(v / v)$ surfactant P20], which was used as both running and regeneration buffer. Data were processed with the Biacore T100 Evaluation software v.2.0.1. Data-collection and model statistics are presented in Table 1. All structures of cetuximab Fab-meditope complexes have been deposited in the RCSB PDB (http://www.rcsb.org) with the following accession codes: CQYDLSTRRLKC (F3Y), 5t1m; CQHDLSTRRLKC (F3H), 5euk; GQQDLSTRRLKG (F3Q), 5i2i; GQ(2-Br-F)DLSTRRLKG [F3(2-BrF)], 5itf; GQ(3-Br-F)DLSTRRLKG [F3(3-BrF)], 5ir1; GQ(4-Br-F)DLSTRRLKG [F3(4-BrF)], 5iop; CQA $(\mathrm{Ph})_{2}$ DLSTRRLKC [F3A(Ph) 2 ], 5t11; CQFDYSTRRLKC (L5Y), 5f88; CQFDESTRRLKC (L5E), 5etu; CQFDQSTRRLKC (L5Q), 5th2; CQFDA(Ph) 2 STRRLKC [L5A(Ph) 2 ], 5t1k; GQFDLST(Cit)RLKG (R8Cit), 5ivz; GQFDLSTR(Cit)LKG (R9Cit), 5iv2; CQFDLSTRRQKC (L10Q), 5 ff6.

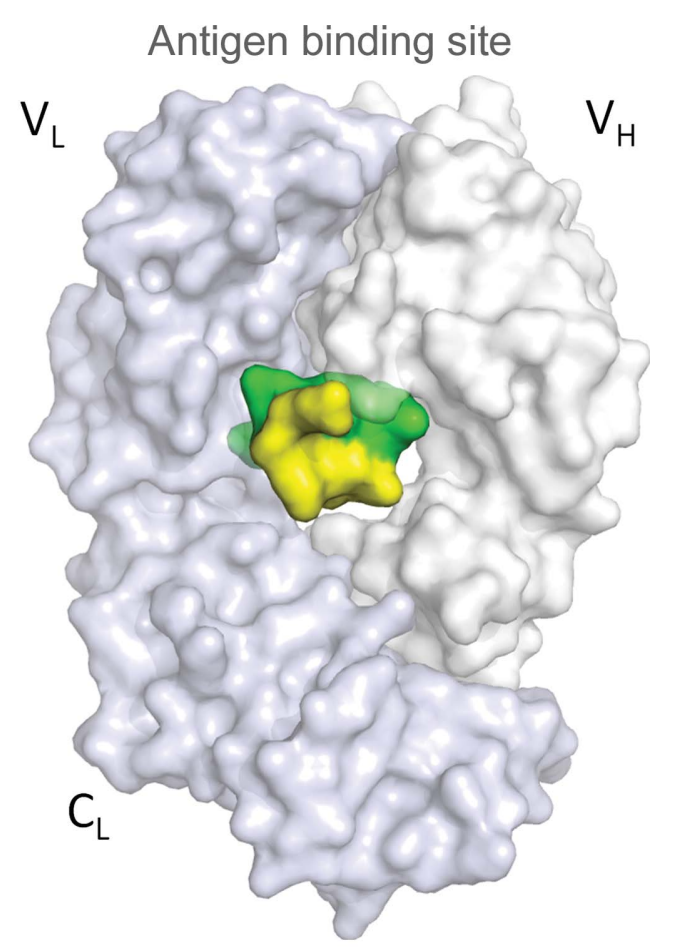

(c)

Figure 1

Meditope binding site. (a) Model of cetuximab IgG based on PDB entry 1igt (Harris et al., 1997). The light chain is shown in light blue and the heavy chain in light gray. $(b, c)$ The cavity in the Fab arm can accommodate the meditope peptide (PDB entry 4gw1; Donaldson et al., 2013). The residues that are under investigation in this report are highlighted in green. 
Table 1

Data-collection and refinement statistics.

Values in parentheses are for the highest resolution shell.

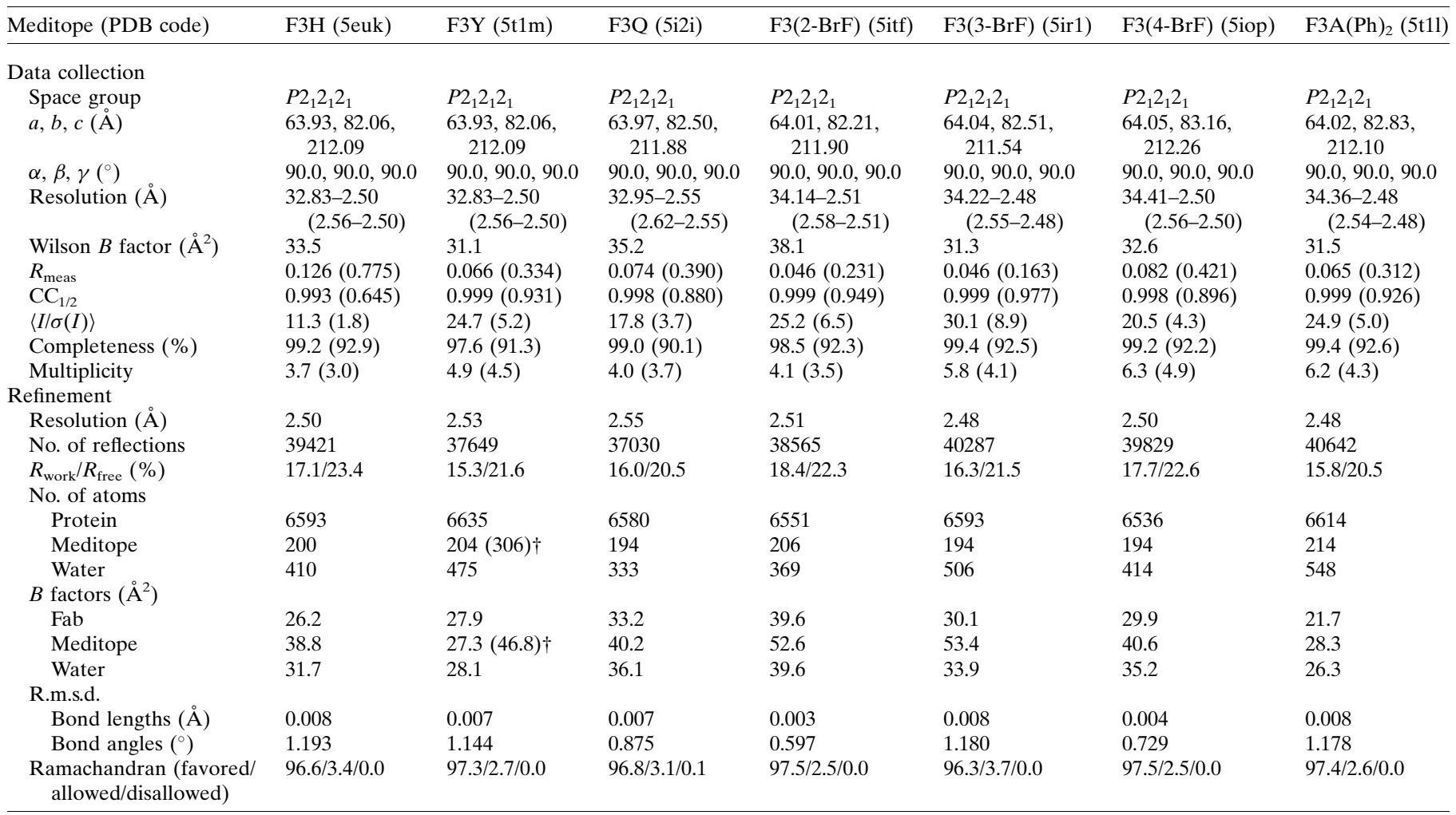

\begin{tabular}{|c|c|c|c|c|c|c|c|}
\hline Meditope (PDB code) & L5Y (5f88) & L5E (5etu) & L5Q (5th2) & $\mathrm{L} 5 \mathrm{~A}(\mathrm{Ph})_{2}(5 \mathrm{t} 1 \mathrm{k})$ & R8Cit (5ivz) & R9Cit (5iv2) & L10Q (5ff6) \\
\hline \multicolumn{8}{|l|}{ Data collection } \\
\hline $\begin{array}{l}\text { Space group } \\
a, b, c(\AA)\end{array}$ & $\begin{array}{l}P 2_{1} 2_{1} 2_{1} \\
64.24,83.14 \\
211.94\end{array}$ & $\begin{array}{l}P 2_{1} 2_{1} 2_{1} \\
64.38,82.87, \\
213.00\end{array}$ & $\begin{array}{l}P 2_{1} 2_{1} 2_{1} \\
64.19,83.12, \\
212.56\end{array}$ & $\begin{array}{l}P 2_{1} 2_{1} 2_{1} \\
64.28,83.25, \\
212.30\end{array}$ & $\begin{array}{l}P 2_{1} 2_{1} 2_{1} \\
64.34,82.57, \\
212.05\end{array}$ & $\begin{array}{l}P 2_{1} 2_{1} 2_{1} \\
64.14,83.19, \\
212.46\end{array}$ & $\begin{array}{l}P 2_{1} 2_{1} 2_{1} \\
64.08,83.05, \\
212.67\end{array}$ \\
\hline$\alpha, \beta, \gamma\left({ }^{\circ}\right)$ & $90.0,90.0,90.0$ & $90.0,90.0,90.0$ & $90.0,90.0,90.0$ & $90.0,90.0,90.0$ & $90.0,90.0,90.0$ & $90.0,90.0,90.0$ & $90.0,90.0,90.0$ \\
\hline Resolution $(\AA)$ & $\begin{array}{l}33.15-2.48 \\
\quad(2.55-2.48)\end{array}$ & $\begin{array}{l}32.65-2.53 \\
\quad(2.60-2.53)\end{array}$ & $\begin{array}{l}44.72-1.84 \\
\quad(1.89-1.84)\end{array}$ & $\begin{array}{l}33.19-2.48 \\
\quad(2.54-2.48)\end{array}$ & $\begin{array}{l}34.29-2.48 \\
\quad(2.54-2.48)\end{array}$ & $\begin{array}{l}33.16-2.48 \\
\quad(2.55-2.48)\end{array}$ & $\begin{array}{l}33.12-2.50 \\
\quad(2.56-2.50)\end{array}$ \\
\hline Wilson $B$ factor $\left(\AA^{2}\right)$ & 29.1 & 32.8 & 33.2 & 31.4 & 26.5 & 24.6 & 217.1 \\
\hline$\langle I / \sigma(I)\rangle$ & $25.2(9.2)$ & $15.7(3.0)$ & $15.0(2.0)$ & 23.5 & $38.1(11.4)$ & $29.1(8.3)$ & $23.5(7.3)$ \\
\hline Completeness (\%) & $95.1(72.3)$ & $99.6(98.1)$ & $92.3(94.6)$ & $99.1(90.3)$ & $98.2(91.1)$ & $99.3(93.2)$ & $99.3(92.6)$ \\
\hline Multiplicity & $5.2(4.6)$ & $3.9(3.4)$ & $3.5(3.3)$ & $4.6(3.2)$ & $6.2(4.2)$ & $5.8(4.1)$ & $4.8(3.7)$ \\
\hline \multicolumn{8}{|l|}{ Refinement } \\
\hline Resolution $(\AA)$ & 2.48 & 2.53 & 1.84 & 2.48 & 2.48 & 2.48 & 2.50 \\
\hline No. of reflections & 39079 & 38822 & 91871 & 41103 & 40196 & 40876 & 39902 \\
\hline$R_{\mathrm{work}} / R_{\text {free }}(\%)$ & $16.3 / 21.6$ & $18.0 / 23.2$ & $16.1 / 18.4$ & $16.0 / 21.3$ & $17.6 / 23.0$ & $16.5 / 21.1$ & $15.5 / 20.1$ \\
\hline \multicolumn{8}{|l|}{$B$ factors $\left(\AA^{2}\right)$} \\
\hline Fab & 27.8 & 28.8 & 30.6 & 20.7 & 20.3 & 22.5 & 28.5 \\
\hline Meditope & 32.5 & 34.9 & 37.0 & 27.3 & 27.2 & 28.4 & 34.4 \\
\hline Water & 32.5 & 32.8 & 44.0 & 25.7 & 26.4 & 30.5 & 33.6 \\
\hline \multicolumn{8}{|l|}{ R.m.s.d. } \\
\hline Bond lengths (Å) & 0.003 & 0.002 & 0.006 & 0.008 & 0.007 & 0.003 & 0.008 \\
\hline Bond angles $\left({ }^{\circ}\right)$ & 0.664 & 0.683 & 0.873 & 1.149 & 0.916 & 0.644 & 1.180 \\
\hline $\begin{array}{l}\text { Ramachandran (favored/ } \\
\text { allowed/disallowed) }\end{array}$ & $97.6 / 2.4 / 0.0$ & $97.0 / 2.9 / 0.1$ & $98.4 / 1.6 / 0.0$ & $97.5 / 2.5 / 0.0$ & $96.7 / 3.3 / 0.0$ & $96.9 / 3.1 / 0.0$ & $96.8 / 3.2 / 0.0$ \\
\hline
\end{tabular}

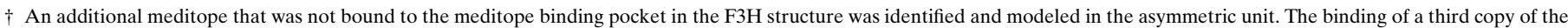

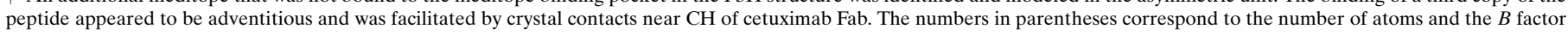
calculated for all three peptides. 


\section{Results and discussion}

To exploit the Fab-meditope interaction for drug delivery, imaging and enhanced internalization, we sought to modulate the affinity of the interaction through the modification of meditope side chains. Moreover, because the meditope peptide consists of only 12 residues, it is straightforward to incorporate non-natural amino acids using routine solid-state peptide-synthetic methods. In our initial discovery and characterization of the interaction, mutagenesis of phenylalanine at position 3 , leucine at position 5 and arginine at position 8 to alanine significantly reduced the affinity of the interaction (Donaldson et al., 2013). Here, we further characterize these sites as well as Arg9 and Leu10. Of the remaining positions, Asp4, Ser6 and Thr7 form a class $3 \beta$-hairpin (Milner-White \& Poet, 1986) and are likely to be important for tertiary structure, while positions 2 and 11 are solvent-exposed and are associated with high $B$ factors. Thus, we did not include these residues in this analysis. We were specifically interested in increasing the half-life of the interaction, which is independent of peptide concentration (note that the dissociation constants, $K_{\mathrm{d}}$, for some variants were difficult to calculate given the difficulty in determining the concentration of some of the meditope variants and we estimate that there is a $38 \%$ error in $K_{\mathrm{d}}$; see Bzymek et al., 2016). Of note, there are two meditopeFab complexes in the asymmetric unit and thus we observe each interaction twice.

\subsection{Substitutions at position 3}

Firstly, we focused on substitutions at position 3. In the original cQFD meditope, we observed that the phenylalanine ring in position 3 stacks against the amide group of Gln39 in the heavy chain (Gln39 $\mathrm{HC}$ ), potentially contributing to the

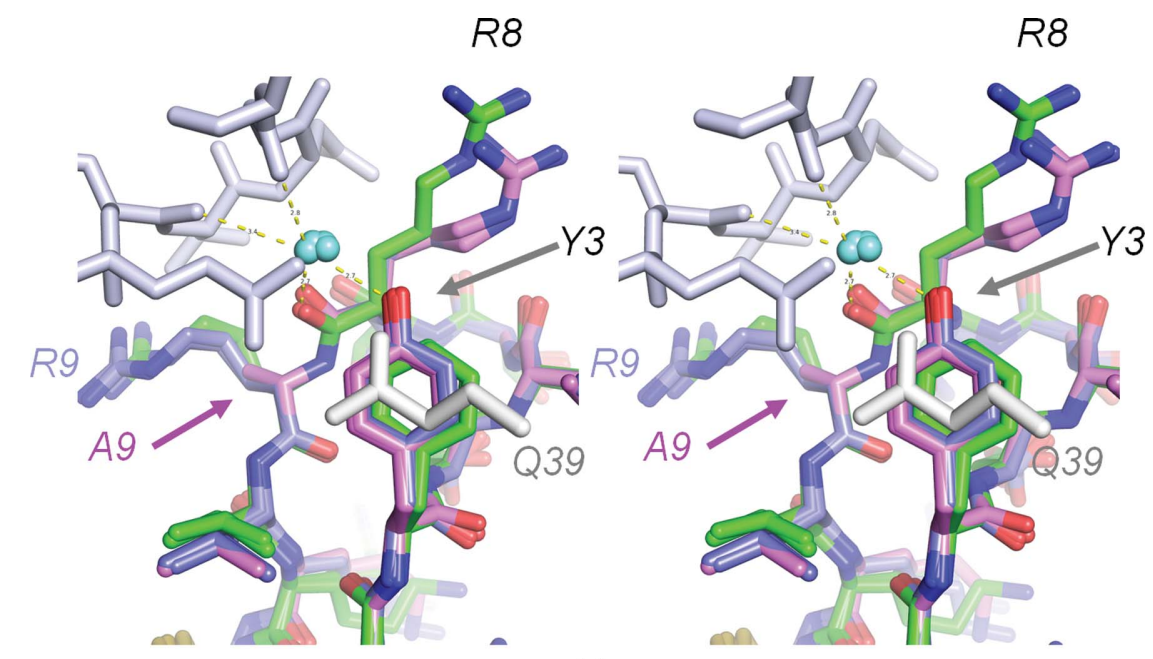

$(a)$
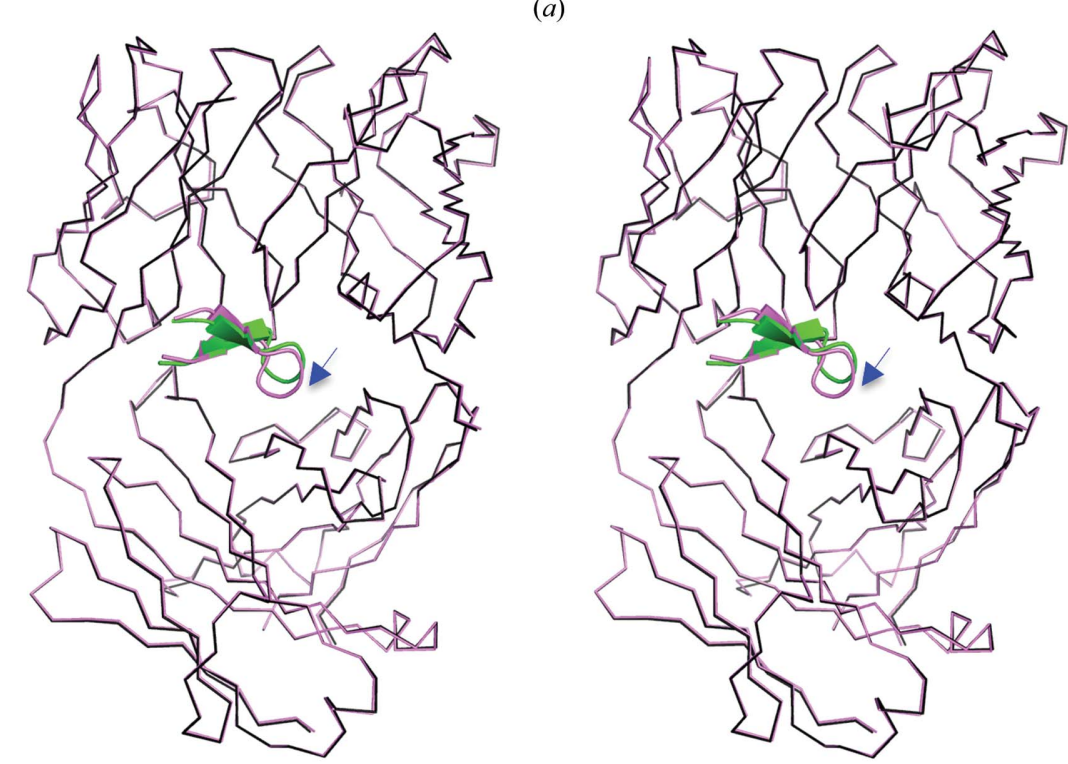

(b)

Figure 2

Tyrosine at position 3 affects Arg8 (stereoviews). (a) Superposition of cQYN (pink C atoms) and F3Y, where the alanine at position 9 (A9) is substituted by $\operatorname{Arg}$ (R9) (light blue C atoms), superimposed on the cQFD meditope (green C atoms). The presence of a hydroxyl from Tyr3 (Y3) sterically occludes the Arg8 side chain, resulting in the loss of a hydrogen bond to the backbone of Gln111 in the heavy chain. The hydroxyl group of Y3, however, leads to the coordination of a water molecule. (b) Superposition of the Fab (cQFD in black and cQYN in pink) shows that the hydroxyl substitution leads to a slight reorientation of the meditope with respect to the Fab. 

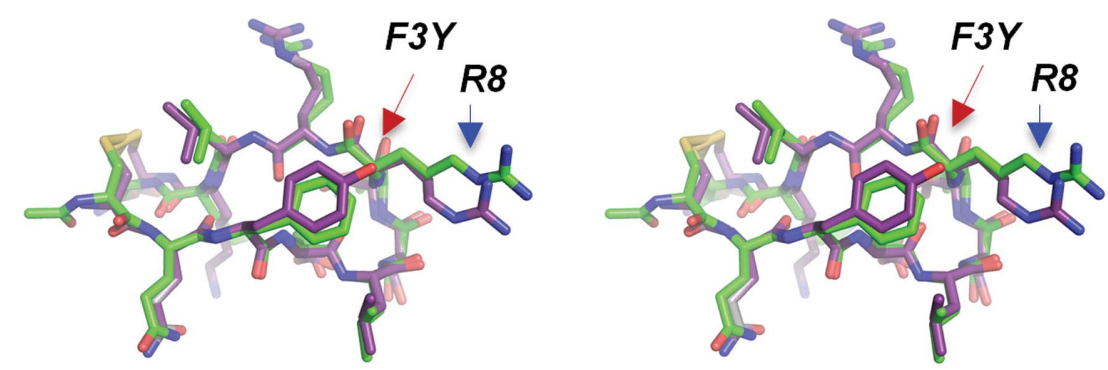

(a)
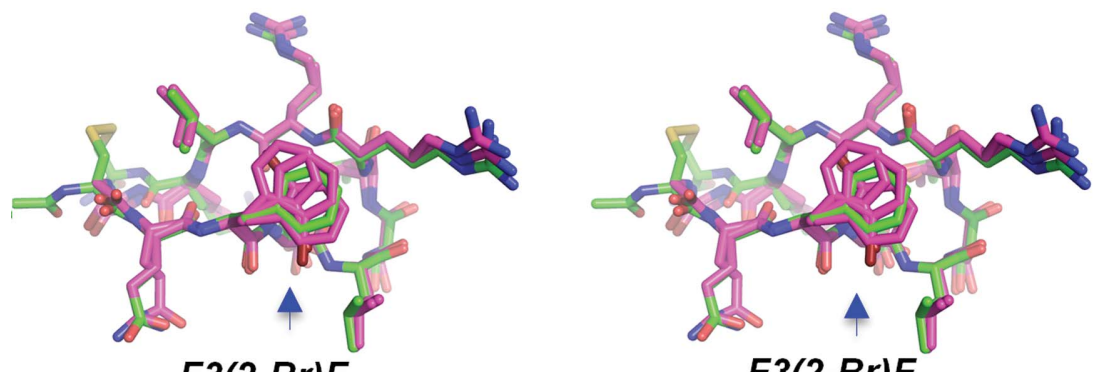

(b)
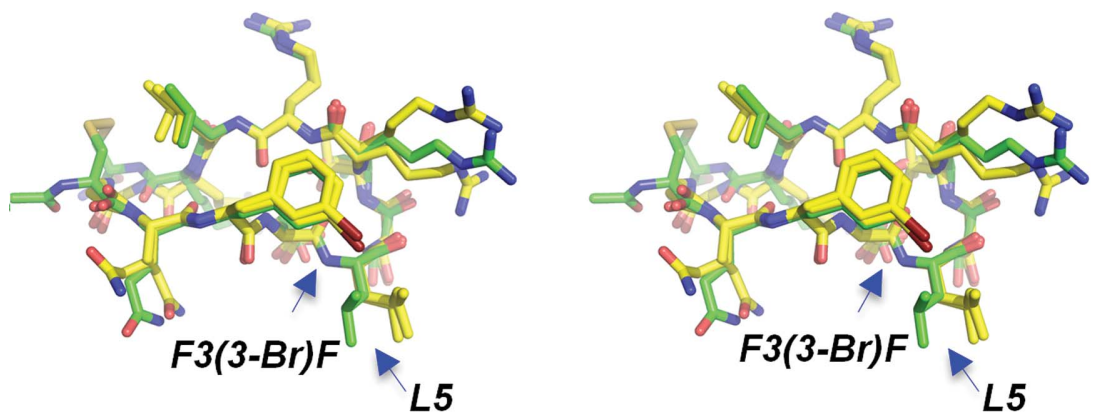

$(c)$
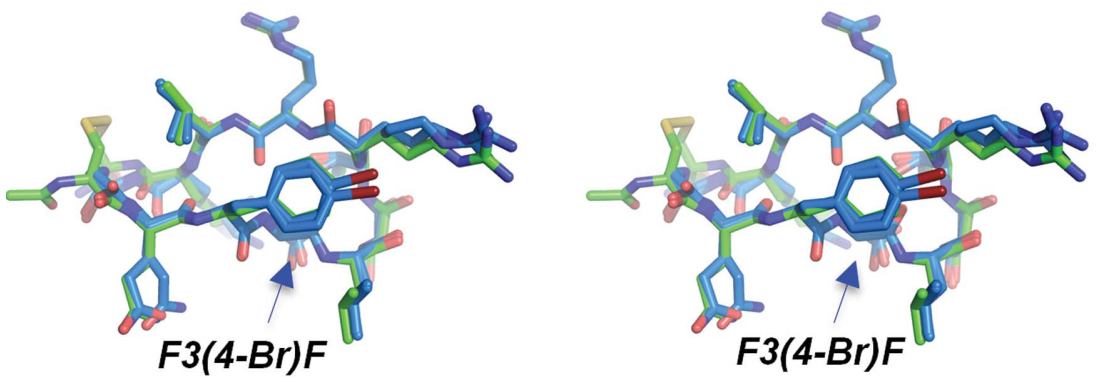

$(d)$
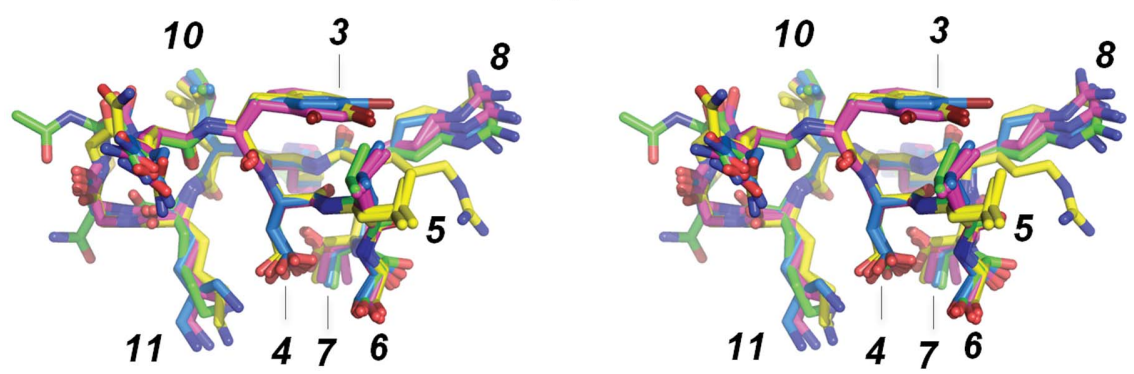

(e)

Figure 3

Substitutions of the phenylalanine at position 3 with brominated phenylalanine analogues, shown in stereo and superimposed on the cQFD meditope (green C atoms). (a) Viewed from the top, tyrosine (purple C atoms) at position 3 blocks the extension of the side chain of Arg8 (R8). (b) Substitution with 2-bromophenylalanine (2-BrF; magenta $\mathrm{C}$ atoms) does not affect the positioning of R8; however, there are multiple conformation of the 2-BrF side chain. (c) Substitution with 3-bromophenylalanine (3-BrF; yellow C atoms) affects R8; however, it also produces a conformational change in Leu5 (L5). (d) Substitution with 4-bromophenylalanine (4-BrF; blue C atoms) slightly perturbs R8. (e) Side view with each variant superimposed on the cQFD meditope. 
Table 2

Binding kinetics for meditope variants to cetuximab.

\begin{tabular}{lllcc}
\hline & & $k_{\mathrm{a}}\left(M^{-1} \mathrm{~s}^{-1}\right)$ & & \\
Meditope & & $\times 10^{4}$ & $k_{\mathrm{d}}\left(\mathrm{s}^{-1}\right)$ & $K_{\mathrm{d}}(\mu M)$ \\
\hline CQFDLSTRRLKC $\dagger$ & (cQFD) & 8.8 & 0.015 & 0.17 \\
GQFDLSTRRLKG $\dagger$ & & 1.7 & 0.083 & 5.0 \\
CQYDLSTRRLKC & F3Y & 8.5 & 0.132 & 1.5 \\
CQQDLSTRRLKC & F3Q & & & $>50 \ddagger$ \\
CQHDLSTRRLKC & F3H & & & $>50 \ddagger$ \\
GQ(2-Br)FDLSTRRLKG & F3(2-Br)F & 15 & 0.270 & 1.8 \\
GQ(3-Br)FDLSTRRLKG & F3(3-Br)F & & & $>5.4 \S$ \\
GQ(4-Br)FDLSTRRLKG & F3(4-Br)F & & & $29 \ddagger$ \\
CQ(4-Br)FDLSTRRLKC & F3(4-Br)F & 3.6 & 0.101 & 2.8 \\
& disulfide & & & \\
CQA(Ph) 2 DLSTRRLKC & F3A(Ph) & 4.5 & 0.011 & 0.24 \\
CQFDESTRRLKC & L5E & 0.34 & 0.444 & 130 \\
CQFDQSTRRLKC & L5Q & 0.46 & 0.148 & 30 \\
CQFDYSTRRLKC & L5Y & & & $>50 \ddagger$ \\
CQFDA(Ph) 2 STRRLKC & L5A(Ph) 2 & 13 & 0.068 & 0.53 \\
GQFDLST(Cit)RLKG & R8(Cit) & & & $>50 \ddagger$ \\
GQFDLSTR(Cit)LKG & R9(Cit) & & & $>8.0 \ddagger$ \\
CQFDLSTRRQKC & L10Q & 4.1 & 0.390 & 9.5 \\
\hline
\end{tabular}

$\dagger$ Data from Bzymek et al. (2016). $\ddagger$ Approximate value $\left(k_{\mathrm{a}}\right.$ and/or $k_{\mathrm{d}}$ are outside the measurement range for the Biacore T100). $\S$ Affinity fit.

overall binding affinity of the meditope through $\pi$-stacking (James et al., 2011). We also observed the same rotamer conformation in a meditope variant, cQYN, bound to the cetuximab Fab (Donaldson et al., 2013). However, the cQYN peptide bound to the Fab with a much lower affinity $\left(K_{\mathrm{d}}=\right.$ $3.5 \mu M$; Donaldson et al., 2013). Superposition of the backbone peptide residues of the cQFD and cQYN variants (but not the Fab chains) indicated that the hydroxyl group of the tyrosine of CQYN sterically interferes with the position of the side chain of Arg8, breaking an electrostatic interaction between the guanidinium group and Gln111 HC observed in the original cQFD-Fab structure (Donaldson et al., 2013). While the loss of this electrostatic bond could account for the difference in affinity, substitution of the tyrosine led to the coordination of a water molecule between the hydroxyl and the backbone carbonyl O atom of Arg39 and Gly42 of the light chain and induced an extended water network between the meditope and the Fab (Fig. 2a). This coordination led to a slight reorientation of the meditope with respect to the Fab (Fig. 2b). The r.m.s.d. between the Fabs is $0.53 \AA$ calculated over $12 \mathrm{C}^{\alpha}$ atoms.

In addition to the substitution of Phe 3 by tyrosine, Arg9 was also substituted with an alanine in the original cQYN meditope. To determine whether the loss of affinity of the cQYN meditope compared with the cQFD meditope was owing to this substitution, we constructed a Phe3Tyr variant of the cQFD peptide. The affinity of this meditope variant, $K_{\mathrm{d}}=$ $1.5 \mathrm{~m} M$, was slightly better than that of the original cQYN $\left(K_{\mathrm{d}}=3.5 \mathrm{~m} M\right.$; Table 2), but remained weaker than that of the original $\mathrm{cQFD}$ peptide. The structure of this meditope variant bound to the Fab was very similar to that of the cQYN meditope (r.m.s.d. of $0.22 \AA$ calculated over $12 \mathrm{C}^{\alpha}$ atoms). As before, the hydroxyl group of Tyr3 sterically blocked the Arg8 side chain from making the backbone hydrogen bond, coordinated a water molecule and was slightly reoriented compared with the original cQFD meditope (Fig. 3a). More- over, the side chain of Arg9 mimicked the same rotamer conformation as found in the cQFD meditope. Thus, the lower affinity is likely to be owing to loss of the electrostatic interaction between the guanidinium of Arg8 and the backbone carbonyl of Gln111 HC caused by the hydroxyl group of Tyr3.

Next, we tested whether the incorporation of a halogen in the phenyl group could afford a halogen-hydrogen bond between the meditope and Tyr87 LC that could increase the affinity (Figs. 3; Voth et al., 2009). The structure with bromine at the ortho (2) position indicated that the aromatic ring lies in the same plane as the phenyl group of Phe3, while Arg8 was able to maintain the backbone hydrogen bond (Fig. $3 b$ ). We observed electron density suggesting two conformations of 2-bromophenylalanine in one of the two meditopes in the asymmetric unit, with one of the $\mathrm{Br}$ atoms $3.2 \AA$ from the $\mathrm{OH}$ of Tyr87 in the light chain (Tyr87 LC; Supplementary Fig. S2). This interaction did not translate to higher affinity; in fact, bromine at the ortho position (2) of the phenyl ring significantly reduced the affinity compared with the original meditope $\left(K_{\mathrm{d}}=1.5 \mathrm{~m} M\right.$; Table 2$)$, but was an improvement over the original meditope cyclized by a diglycine linker $\left(K_{\mathrm{d}}=5 \mathrm{mM}\right.$; Bzymek et al., 2016).

Next, we placed bromine at the meta position (3), again to test whether it could interact with the hydroxyl of Tyr87. The structure revealed that the bromine points away from Tyr87, forcing the side chain of Leu5 in the meditope to adopt a different conformation which is not observed in any of the complexes with other meditope variants (Fig. 3c). This repositioning of the Leu5 side chain also produced a shift of the entire meditope with respect to the Fab. Moreover, greater disorder was observed in the side chain of Arg8 of one of the meditopes in the asymmetric unit ( $B$ factor of $64.8 \AA^{2}$ compared with $51.2 \AA^{2}$ ). The binding affinity was also reduced (Table 2).

Finally, we also produced and characterized a 4-bromophenylalanine variant of the phenylalanine at position 3 . Surprisingly, in one meditope-Fab complex in the asymmetric unit the Arg8 side chain is in an extended conformation similar to that in the original meditope (Fig. $3 d$ ). The guanidinium in the 4-bromophenylalanine variant makes similar hydrogen bonds as in the original meditope (the bond distances $\mathrm{NH} 1 / \mathrm{NH} 2 \cdots \mathrm{O}=\mathrm{C}$ are 3.1/2.9 $\AA$ and 2.6-2.8/2.8$2.9 \AA$, respectively). In the other complex, the Arg8 side chain is not extended, placing the guanidinium group distant from the carbonyl (bond distances $\mathrm{NH} 1 / \mathrm{NH} 2 \ldots \mathrm{O}=\mathrm{C}$ of $3.3 / 4.0 \AA$ ). Of note, the coordinated water that was observed in the tyrosine variants is not observed in either complex of the 4-bromophenylalanine variant. This loss could be related to an unfavorable geometry owing to a slightly longer bond $[\mathrm{C}-\mathrm{Br}$ versus $\mathrm{C}-\mathrm{O}(\mathrm{H})]$. On the other hand, the bromine in 4-bromophenylalanine is 3.7 and $3.9 \AA$ from the hydroxyl of Tyr94 HC, forming a weak halogen-hydrogen bond (Voth et al., 2009; Fig. 3d). While the contribution of each of these potential explanations is unclear, the bromine is positioned such that it does not interfere with the side chain of Arg8. Based on these observations, we anticipated that the affinity of this meditope variant would be higher; however, it was in fact 
weaker $\left(K_{\mathrm{d}}=2.8 \mu M\right.$ for the disulfide-bonded peptide) compared with the Phe3Tyr meditope $\left(K_{\mathrm{d}}=1.2 \mu M\right.$; Table 2$)$. Of note, the structure was solved with a diglycine-linked peptide, which also showed reduced affinity for cetuximab ( $K_{\mathrm{d}}=29 \mu M$ versus $5 \mu M$ for the respective diglycine-linked meditope (Table 2; Bzymek et al., 2016).

To test whether other side chains could be substituted at position 3 to form additional bonds to the Fab and/or through the water network, we used PyMOL (Schrödinger) to identify side chains and different rotamers and to replace the phenylalanine with a glutamine and a histidine. The histidine side chain is aromatic but is capable of acting as a proton acceptor or donor (depending on the $\mathrm{pH}$ ). Also, the modelled rotamers placed the glutamine side chain within a suitable distance from several proton donors/acceptors for the formation of hydrogen bonds.

While the crystal structure of the glutamine meditope variant shows significant disorder for the glutamine side chain (Fig. 4a), the imidazole side chain of the histidine variant adopts a different rotamer from that of the original phenylalanine (Fig. $4 b$ ). The imidazole ring is rotated $\sim 170^{\circ}$ around the $\mathrm{C}^{\alpha}-\mathrm{C}^{\beta}$ bond, losing the potential $\pi$-stacking with the highly conserved Gln-Gln interaction between the light and heavy chains. Rather, the imidazole makes weak interactions with the backbone carboxyl $\mathrm{O}$ atom of Ala100 LC $\left(d_{\mathrm{NE} 2 \cdots \mathrm{O}=\mathrm{C}}\right.$ of $\sim 3.1-3.2 \AA)$ and its amide $\mathrm{N}$ atom $\left(d_{\mathrm{NH} \cdots \mathrm{ND} 1}\right.$ of $\left.3.0-3.3 \AA\right)$ (Fig. $4 b$ and Supplementary Fig. S3). We note that the $\mathrm{pH}$ of the crystallization buffer of 5.5 (which is close to the $\mathrm{p} K_{\mathrm{a}}$ of the imidazole ring of histidine) should have afforded a mostly protonated form of histidine. We also note that at the $\mathrm{pH}$ value of 7.4 used for SPR experiments over $90 \%$ of histidine (assuming that the $\mathrm{p} K_{\mathrm{a}}$ of a free histidine imidazole side chain is 6.0) would be in its unprotonated form, potentially weakening this interaction. SPR measurements indicate that the variants exhibited weaker binding to the Fab than the original meditope (Table 2).

Superposition of the F3H complex onto the cQFD complex, however, suggested that it may be possible to increase the affinity by introducing a $\beta$-branched, unnatural amino acid containing a phenyl group. Owing to its commercial availability, we incorporated diphenylalanine at position 3. The crystal structure of this variant indicated that the two phenyl rings mimic the phenyl and imidazole rings in the original and the F3H structures (Fig. 4c), with no significant changes in the
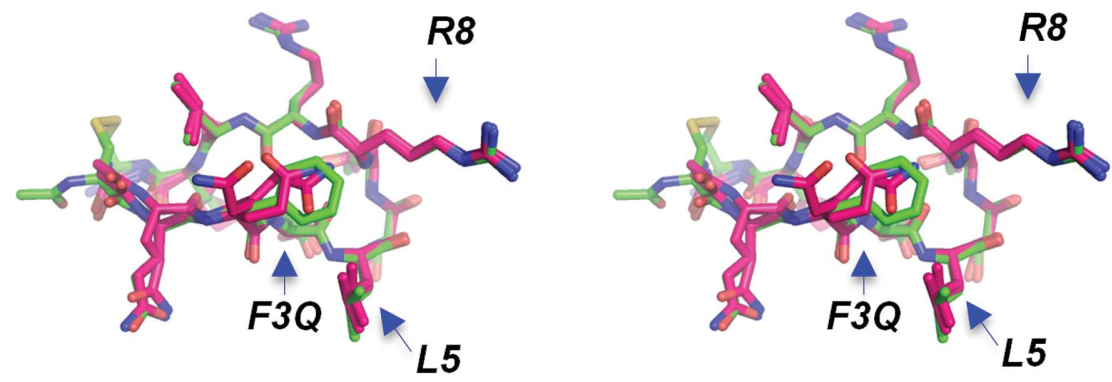

(a)
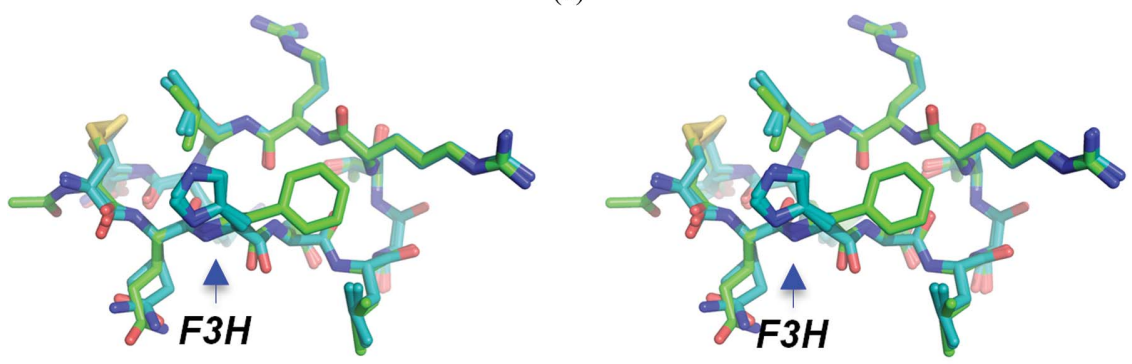

(b)
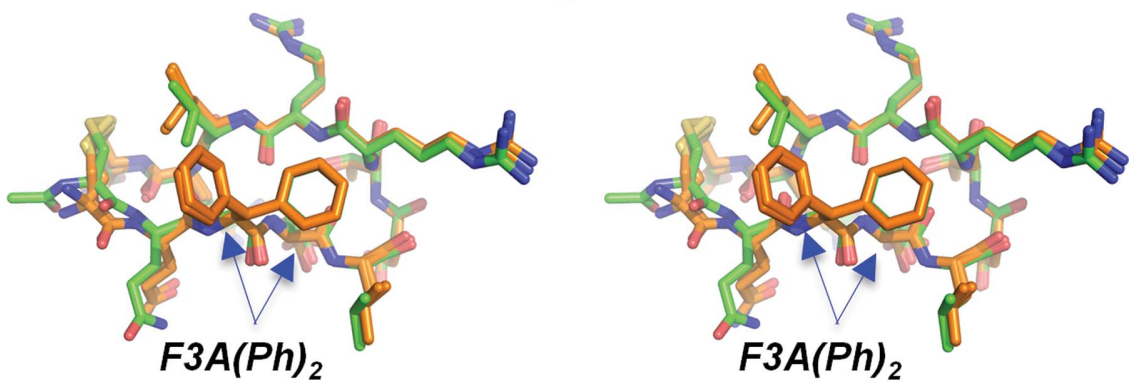

(c)

Figure 4

Substitutions of the phenylalanine at position 3, shown in stereo and superimposed on the cQFD meditope (green C atoms). (a) Substitution of phenylalanine with glutamine led to multiple side-chain rotamers (hot pink $\mathrm{C}$ atoms). (b) Substitution of phenylalanine with histidine led to a single conformation exposed to the solvent (cyan C atoms). (c) Based on these observations, we substituted phenylalanine with diphenylalanine (orange $\mathrm{C}$ atoms). One phenyl group of the diphenylalanine substitution superposed with the phenyl ring of the cQFD meditope. The other phenyl group superposed well with the imidazole ring of the histidine meditope variant. 

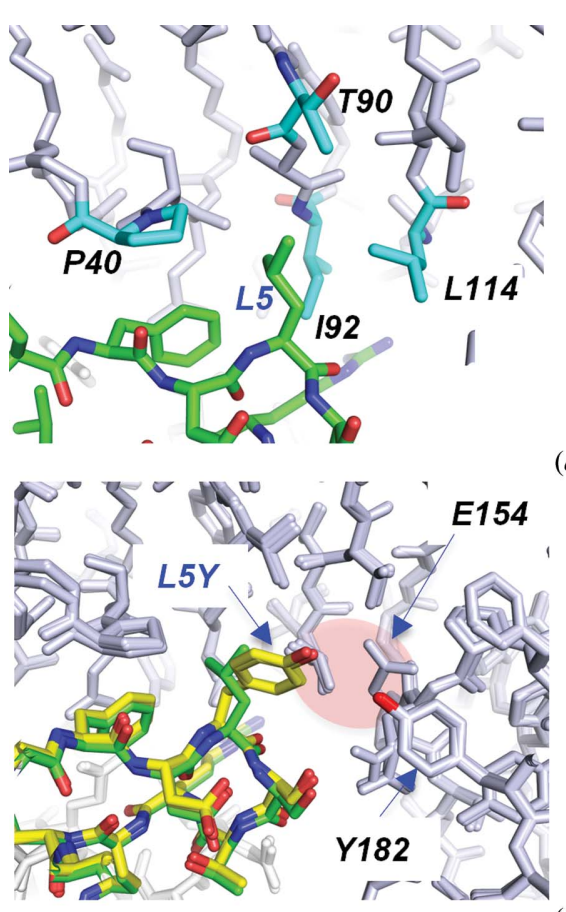

(b)
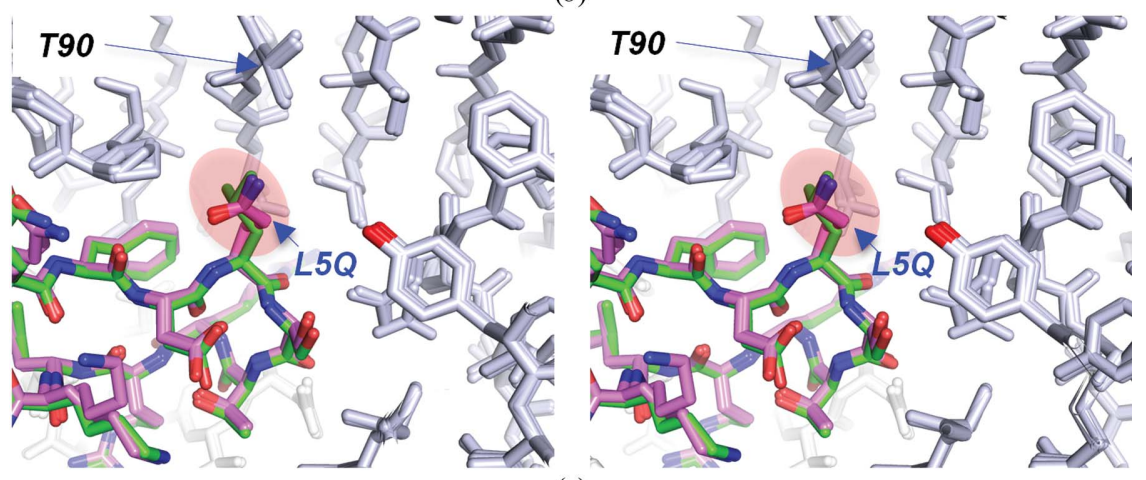

(c)
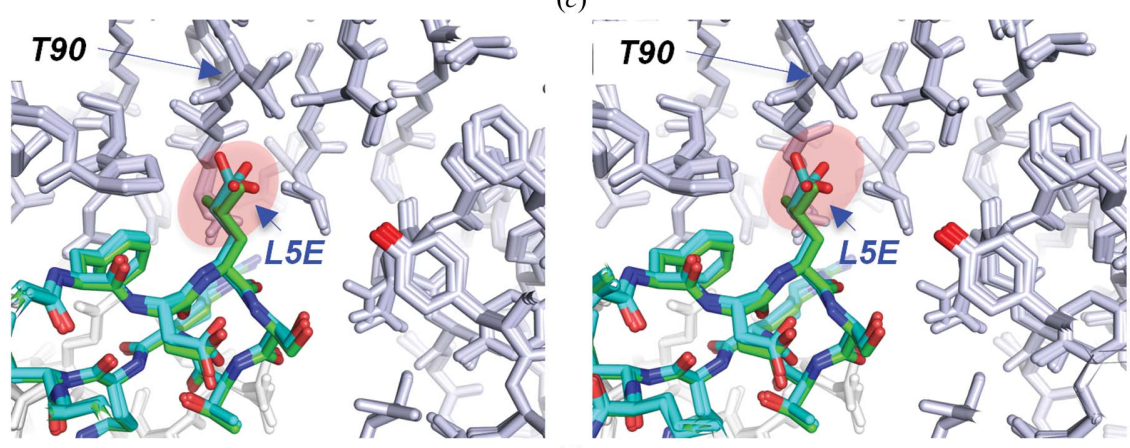

(d)
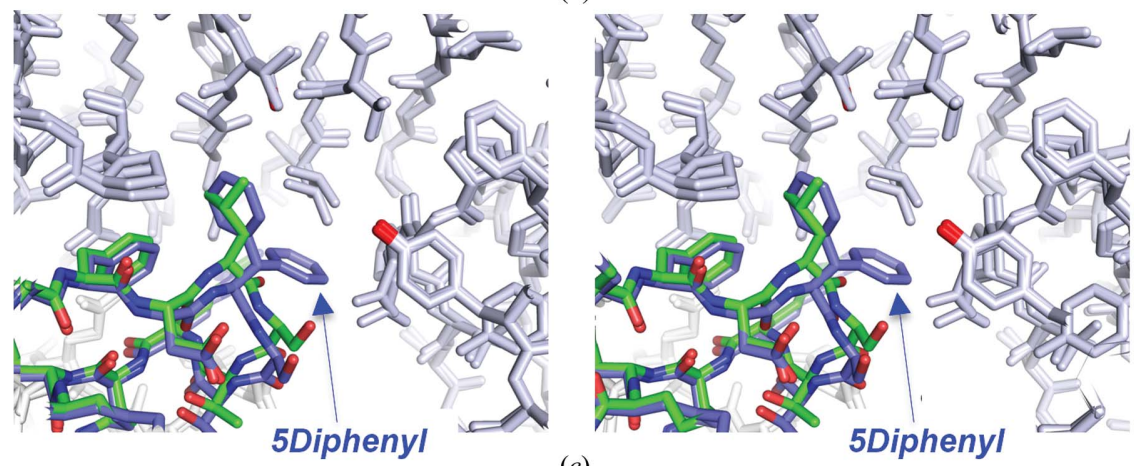

overall tertiary structure (r.m.s.d. of $0.6 \AA$ calculated over $12 \mathrm{C}^{\alpha}$ atoms). Unlike the other substitutions at the F3 position, the affinity of this variant for cetuximab is similar to that of the original meditope $\left(K_{\mathrm{d}}=\right.$ $0.24 \mu M$ versus $0.17 \mu M$, respectively) with an off-rate that is approximately $40 \%$ slower. While the concentration of the peptides was difficult to determine owing to the low extinction coefficient (Bzymek et al., 2016), off-rates are independent of concentration and thus comparison of the off-rates is more accurate. Given this, every substitution at position $\mathrm{F} 3$ significantly increased the off-rate (e.g. nearly tenfold) except for diphenylalanine. We speculate that the affinity could be further improved using a $\beta$ branched amino acid in which the exposed phenyl group is hydrophilic and capable of making hydrogen bonds to the Fab.

\subsection{Substitutions at position 5}

Next, we turned our attention to leucine at position 5 in the meditope peptide. Leu5 resides in a relatively hydrophobic pocket lined with Pro41 HC, Thr90 HC, Ile92 HC and Leu114 HC (Fig. 5a). In an attempt to retain hydrophobic interactions and create additional interactions through hydrogen bonds, we substituted the leucine with a tyrosine (Fig. 5b). The hydroxyl group forms a weak hydrogen bond to the hydroxyl group of Tyr182 HC (3.6/3.8 $)$ ). In addition, a rotamer of Glu154 HC observed in one of the complexes in the asymmetric unit forms

Figure 5

Substitutions of leucine at position 5, shown in stereo and superimposed on the cQFD meditope (green $\mathrm{C}$ atoms). ( $a$ ) The leucine side chain resides in a hydrophobic pocket defined by Thr90 (T90), Ile92 (I92) and Leu114 (L114) of the Fab heavy chain and Pro40 (P40) of the Fab light chain. (b) Substitution of leucine with tyrosine in the meditope positions the hydroxyl group near the side chain of Glu154 (E154) and the hydroxyl group of Tyr182 (Y182). (c) The replacement of leucine with glutamine at position 5 was intended to create a hydrogen bond to the hydroxyl group of Y182 in the heavy chain or the hydroxyl of T90 in the light chain. However, the side chain points away from the Fab. (d) Substitution with glutamic acid resulted in positioning of the carboxylic acid in the hydrophobic pocket. The high $B$ factors of the carboxylate suggest that the positioning of the side chain is adventitious. (e) Substitution at position 5 with diphenylalanine places one phenyl group at the same position as the leucine side chain. The other phenyl group extends further into the meditope cavity that is lined with the hydrophobic residues. 
an electrostatic bond to the tyrosine hydroxyl $\left(d_{\mathrm{OH} \cdots \mathrm{OE} 2}=\right.$ $3.4 \AA$ ). The same OE2 of Glu154 HC is within $2.6 \AA$ of OG1 of Thr116 HC. The other rotamer of Glu154 is at a distance of $2.3 \AA$ from OG of Ser6 of the meditope compared with the respective OG of Ser6 of the NCS-related meditope (2.9 $\AA$ ). Despite an additional hydrogen-bonding network, the affinity of this mutant, $K_{\mathrm{d}}>50 \mu M$, is over two orders of magnitude weaker compared with that of the original meditope (Table 2).

In a second effort to create favorable hydrogen-bonding interactions, Leu5 was substituted with glutamine and, separately, glutamate. Based on the few available cetuximab structures at the time, we anticipated that the hydroxyl of Thr90 could be oriented to favor interactions with the carbonyl $\mathrm{O}$ atom of a glutamine or glutamic acid. We also noticed an extended water network in an apo structure solved in-house; upon binding of the L5E/Q meditope, the waters could facilitate interactions between the meditope and Tyr182. Substituting Leu5 with the polar but uncharged glutamine reduced the affinity $\sim 200$-fold, despite the absence of direct interactions between the amide of glutamine and cetuximab
Fab (Fig. 5c). Substitution with glutamate produced a weak hydrogen bond between OE2 of Glu5 and the peptide $\mathrm{NH}$ of Ala91 HC ( $\left.d_{\mathrm{OE} 2 \cdots \mathrm{NH}}=3.1-3.2 \AA\right)$. However, unsurprisingly there are fewer hydrophobic interactions (Fig. 5c). Unfavorable repulsive electrostatic forces between glutamate in position 5 and Glu154 HC are likely to be responsible for the low affinity and the fast off-rate, which is $\sim 30$ times that of the original meditope. The lack of productive interactions with the Fab resulted in a drastic $\sim 760$-fold drop in affinity compared with the original meditope. In contrast, the uncharged glutamine variant has an off-rate that is only approximately nine times faster that of the original meditope.

Finally, we substituted Leu5 with diphenylalanine in an effort to increase hydrophobic interaction between the meditope and Fab. The structure of the diphenylalanine substitution shows that one phenyl ring is in a similar position to the leucine side chain, while the other phenyl group contacts Ile92, Leu114 and Pro155. There is a slight shift in the backbone residues to accommodate the bulky diphenylalanine. The affinity for this mutant was slightly reduced
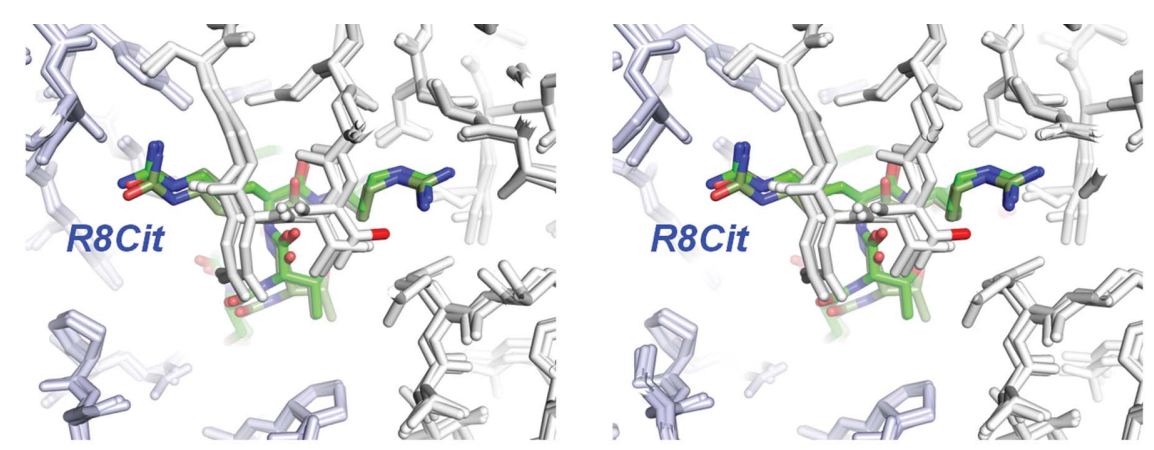

(a)
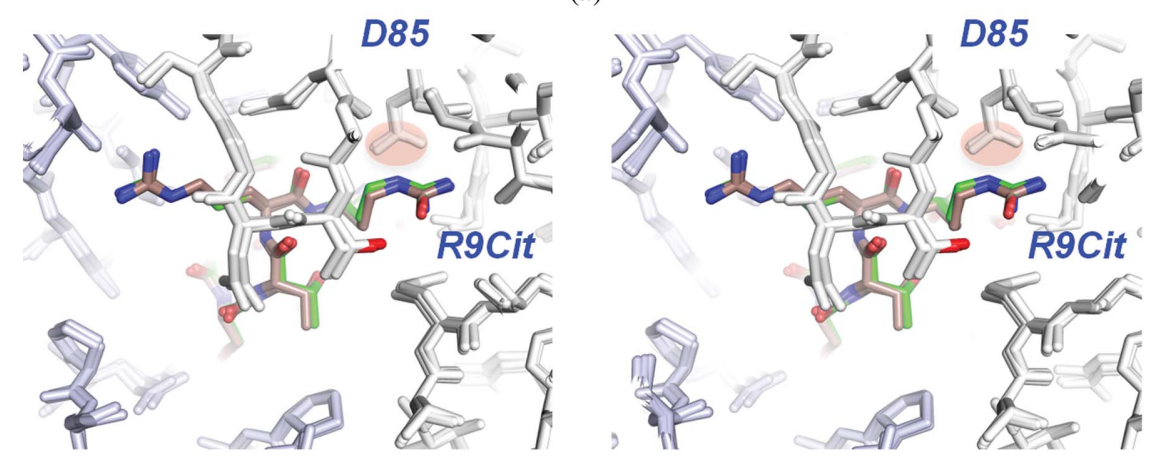

(b)
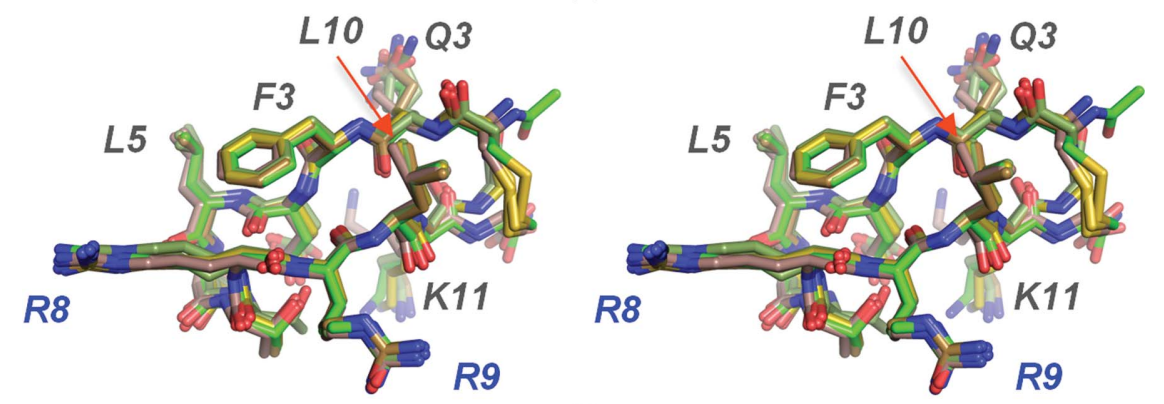

(c)

Figure 6

Citrulline substitutions, shown in stereo and superimposed on the cQFD meditope. The substitution of arginine with citrulline at either $(a)$ position 8 or (b) position 9 gave structures that were indistinguishable from that of the original meditope $(c)$. 

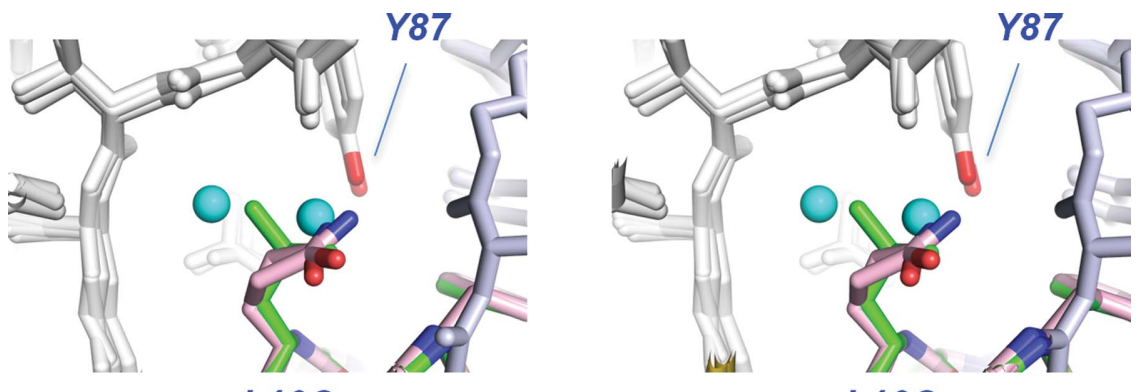

$L 10 Q$

L10Q

Figure 7

Substitutions of leucine at position 10, shown in stereo and superimposed on the cQFD meditope. Leu10 packs against a shallow hydrophobic pocket. Glutamine was substituted for Leu10 in an effort to form a hydrogen bond to the hydroxyl group of Tyr87 adjacent to the hydrophobic pocket. The cyan spheres represent two water molecules present in the apo structure (PDB entry 1yy8).

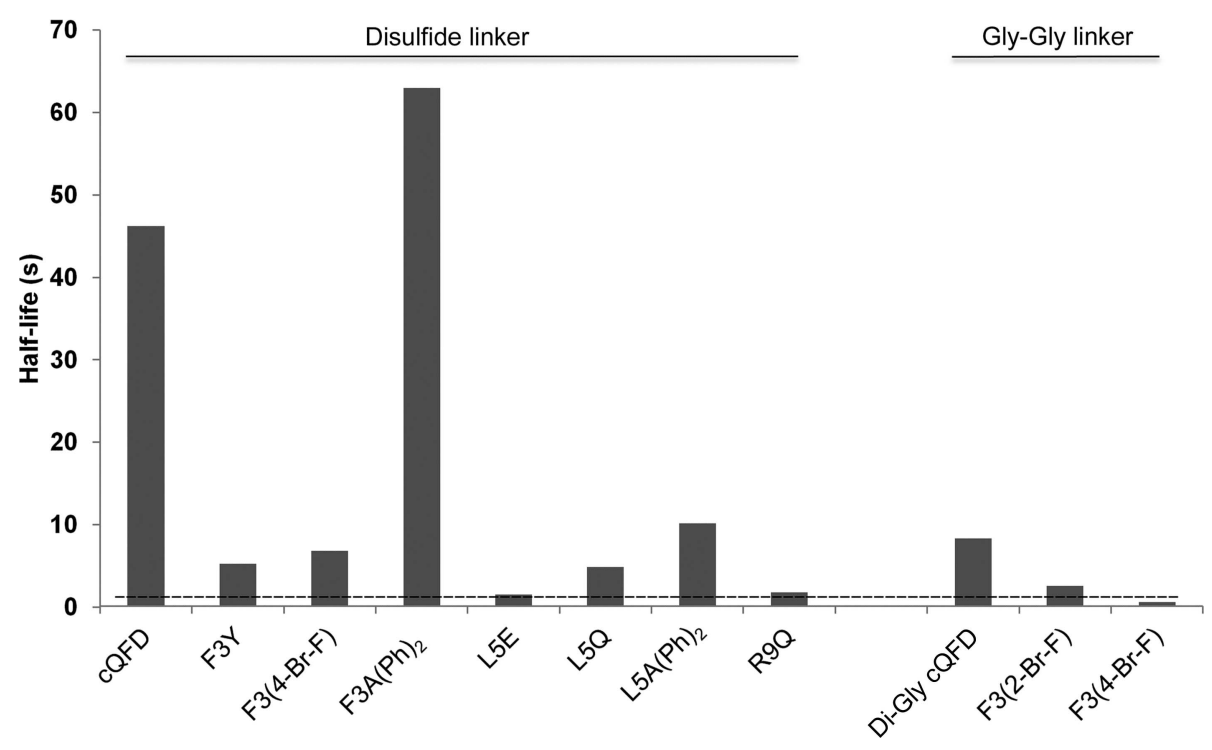

Figure 8

Half-lives of meditope variant-cetuximab interactions. While the on-rate of a bimolecular interaction is dependent on concentration, the off-rate is not. The half-life is related to the off-rate through $t=\ln (2) / k_{\mathrm{d}}$. The dashed line represents the lower limit on the determination of $k_{\mathrm{d}}\left(0.5 \mathrm{~s}^{-1}\right.$, corresponding to a $1.4 \mathrm{~s}$ half-life.) Note that several of the variants were cyclized through a diglycine linker, of which GQ(2-Br-F)DLSTRRLKG [F3(2-BrF)] and GQ(4Br-F)DLSTRRLKG [F3(4-BrF)] allowed the determination of kinetic constants.

compared with the original meditope: $K_{\mathrm{d}}=0.53 \mu M$. However, it was not as severe as the other substitutions, and the off-rate increased approximately fivefold compared with the original meditope.

\subsection{Substitutions at positions 8 and 9}

As arginine side chains carry a positive charge at physiological $\mathrm{pH}$, we tested the effect of substitution of the arginines with noncharged amino acids on binding to cetuximab Fab. Changes in the structure were minimal compared with the original diglycine-linked meditope (r.m.s.d.s of 0.131 and $0.125 \AA$ for Arg8Cit and Arg9Cit, respectively, calculated over $12 \mathrm{C}^{\alpha}$ atoms; Fig. 6). Similar to the Arg8Ala modification, Arg8Cit showed reduced affinity for the meditope $\left(K_{\mathrm{d}}>\right.$ $50 \mu M$ versus $K_{\mathrm{d}}=5 \mu M$ for the arginine diglycine-linked peptide; Bzymek et al., 2016). Beyond a difference in charge, the substitution of the amide for the guanidinium group also reduces the number of hydrogen bonds. There is one hydrogen bond between $\mathrm{NH}_{2}$ (the amide $\mathrm{O}$ atom in citrulline) and $\mathrm{O}=\mathrm{C}$ of Gln111 HC (Donaldson et al., 2013). Similarly, substitution of Arg9 with citrulline resulted in slightly weaker binding with $K_{\mathrm{d}}>8 \mu M\left(K_{\mathrm{d}}=5 \mu M\right.$ for the arginine diglycine-linked peptide; Bzymek et al., 2016); the loss of affinity can be at least in part attributed to the loss of positive charge upon the substitution. The guanidinium group of Arg9 makes contacts with the carboxyl group of Asp85 from the light chain (Donaldson et al., 2013). Thus, the charge from the guanidinium groups plays an important role in the overall affinity.

\subsection{Substitutions at position 10}

Leu10 resides in a relatively polar environment, and upon binding to the Fab it displaces three water molecules bound to the peptide backbone (Fig. 7). We substituted this side chain with the amide of glutamine in an effort to recapitulate the lost hydrogen-bonding interactions. While we observe that the amide $\mathrm{O}$ atom of Gln 10 was $2.9 \AA$ away from the hydroxyl of 
Tyr87, this seemingly productive interaction did not improve the affinity $\left(K_{\mathrm{d}}=9.5 \mu M\right)$ or the half-life of the complex (Table 2), showing a preference for hydrophobic side chains in this environment (Fig. 8).

\section{Conclusion}

Our previous study focused on a cyclization strategy for the meditope peptide and its effect on the affinity for cetuximab Fab (Bzymek et al., 2016). We determined that a disulfidelinked peptide exhibited the best kinetics in terms of the slowest off-rate $\left(k_{\mathrm{d}}=0.015 \mathrm{~s}^{-1}\right)$. Here, we present a detailed structure-binding analysis of multiple side-chain meditope variants of cetuximab Fab. We confirmed that hydrophobic interactions are preferred at positions 3, 5 and 10 of the meditope. Adding polar or charged side chains at those positions resulted in diminished binding, despite the presence of additional hydrogen bonds to the Fab framework. Despite the absence of a gain in binding affinity, the alternate rotamer observed for the histidine substitution at position 3 motivated the diphenylalanine substitution, which increased the lifetime of the interaction (Fig. 8). Attempts to reduce the charge of the residues in positions 8 and 9 resulted in weaker affinity. Interestingly, the change had a larger effect on Arg8 than Arg9, despite the fact that Arg9 forms a salt bridge with Asp85 of the cetuximab light chain. We are currently investigating substitutions on the antibody framework in an attempt to increase the affinity of the meditope-Fab interaction.

\section{Acknowledgements}

JCW acknowledges support from the Alicia and John Kruger Gift, the Leo and Anne Albert Charitable Trust, the Carl and
Roberta Deutsch Fundation and Grant No. R21 CA135216 from NCI. We also acknowledge the Drug Discovery and Structural Biology Core, which is supported by Grant No. P30 CA033572 from the NCI. We also thank the Molecular Observatory of Caltech as well as the SSRL beamline 12-2 scientists for help with X-ray data collection. We thank the current and former members of the Williams laboratory and Dr Gagandeep Singh for helpful insights and discussions.

\section{References}

Bhakta, S., Raab, H. \& Junutula, J. R. (2013). Methods Mol. Biol. 1045, 189-203.

Bzymek, K. P., Ma, Y., Avery, K. A., Horne, D. A. \& Williams, J. C. (2016). Acta Cryst. F72, 434-442.

Chari, R. V. J. (2008). Acc. Chem. Res. 41, 98-107.

Donaldson, J. M., Zer, C., Avery, K. N., Bzymek, K. P., Horne, D. A. \& Williams, J. C. (2013). Proc. Natl Acad. Sci. USA, 110, 17456-17461. Ducry, L. \& Stump, B. (2010). Bioconjug. Chem. 21, 5-13.

Emmons, C. \& Hunsicker, L. G. (1987). Iowa Med. 77, 78-82.

Harris, L. J., Larson, S. B., Hasel, K. W. \& McPherson, A. (1997). Biochemistry, 36, 1581-1597.

James, W. H., Buchanan, E. G., Guo, L., Gellman, S. H. \& Zwier, T. S. (2011). J. Phys. Chem. A, 115, 11960-11970.

Milner-White, E. J. \& Poet, R. (1986). Biochem. J. 240, 289-292.

Rabuka, D., Rush, J. S., deHart, G. W., Wu, P. \& Bertozzi, C. R. (2012). Nature Protoc. 7, 1052-1067.

Suzuki, M., Kato, C. \& Kato, A. (2015). J. Toxicol. Pathol. 28, 133-139.

Trail, P. A. \& Bianchi, A. B. (1999). Curr. Opin. Immunol. 11, 584-588.

Voth, A. R., Khuu, P., Oishi, K. \& Ho, P. S. (2009). Nature Chem. 1, 74-79.

Weiner, L. M., Murray, J. C. \& Shuptrine, C. W. (2012). Cell, 148, 1081-1084.

Wu, A. M. \& Senter, P. D. (2005). Nature Biotechnol. 23, 1137-1146. 